\title{
Alles was Recht ist
}

\section{Unterbliebene Amniozentese}

\author{
Wenn erst postpartal Fehlbildungen erkannt werden, wird von Eltern \\ oft hinterfragt, ob man dies nicht hätte früher sehen können. Mögliche \\ Konsequenz sind mitunter erhebliche Schadensersatzforderungen.
}

V orliegend machten die Eltern zahlreiche Schadensersatzansprüche bei einer Trisomie 21 geltend. Die Patientin befand sich in der 21. SSW, als ihre Ärztin beim Fetus einen beidseitigen Nierenstau feststellte und sie zur Abklärung eventueller Fehlbildungen zum Organ-Ultraschall an den beklagten Kollegen mit Praxis für Pränataldiagnostik überwies. Vor dessen Untersuchung unterschrieb die Patientin einen Anamnese- und Aufklärungsbogen, in dem auf die Möglichkeiten und Grenzen der Ultraschalldiagnostik eingegangen wurde. Infolge der Untersuchung wurde letztlich keine Amniozentese mehr gemacht. Die Gründe dafür waren aber streitig, sodass dem Arzt später vorgeworfen wurde, eine nach Ansicht der Kläger unbedingt indizierte Amniozentese pflichtwidrig unterlassen und die Patientin unzureichend über Untersuchungsmöglichkeiten aufgeklärt zu haben; bei adäquater Diagnostik wäre das Down-Syndrom früher festgestellt und die Schwangerschaft noch abgebrochen worden.

\section{So sah das Gericht den Fall}

Die Klage wurde nach Einholung eines Gutachtens, ergänzender Anhörung des Sachverständigen und Anhörung der Parteien vom LG Dresden (Urteil v. 13.1.2017, 6 O 3129/14) abgewiesen. Weder ein Behandlungs-, noch Aufklärungsoder Beratungsversäumnis war feststellbar. Der Behandlungsfehler wurde im Gutachten nicht bestätigt, da bei der Ultraschalluntersuchung nur eine geringe Pyelektasie (Erweiterung der beiden Nierenbecken auf 3,3 mm links und 3,5 $\mathrm{mm}$ rechts) bei sonst unauffälligem, zeitgerecht entwickeltem Fetus (Herz, Schädel etc. unauffällig) zu sehen gewesen sei und bezüglich der Nieren fachgerecht eine postpartale Kontrolle empfohlen worden sei. Kein weiteres Organ war auffällig und weder ein klarer Soft-, noch Hardmarker lag als Hinweis auf ein DownSyndrom vor. Hinsichtlich der Erweiterung des Nierenbeckens zähle unter anderem erst eine solche über $5 \mathrm{~mm}$ neben anderen Auffälligkeiten zu Softmarkern chromosomaler Anomalien. Das isolierte Auftreten eines allenfalls grenzwertig diskutierten, einzelnen Softmarkers stelle ein vernachlässigbares Risiko dar. Selbst bei Annahme eines isolierten Softmarkers im Low-Risk-Kollektiv ohne weitere Risikofaktoren stelle dies zumindest keine zwingende Indikation für invasive Pränataldiagnostik dar. Vor dem Hintergrund sei auch keine Fehlberatung gegeben. Entgegen der Ansicht der Kläger habe der Beklagte eine Fruchtwasserpunktion nicht als medizinisch geboten anraten müssen. Im Übrigen hatte die Patientin unstreitig den Aufklärungsbogen unterschrieben, sodass sie über die Grenzen der Ultraschalluntersuchung im Klaren war, und unmissverständlich erklärt wurde, dass aus einem unauffälligen Ultraschall nicht mit Sicherheit abgeleitet werden könne, dass ein Kind normal entwickelt und gesund sei. Der Beklagte hatte zudem nachvollziehbar geschildert, dass er sich immer erst das Kind anschaue und danach Ergebnis und Empfehlung mitteile. Dabei werde über das Risiko weiterer Untersuchungen ebenso wie über das Risiko einer Erkrankung gesprochen und nach gemeinsamer Abwägung in der Regel das geringere Risiko gewählt. Vorliegend habe er aufgrund des Befundes wohl gesagt, dass keine signifikanten Auffälligkeiten zu sehen seien, er

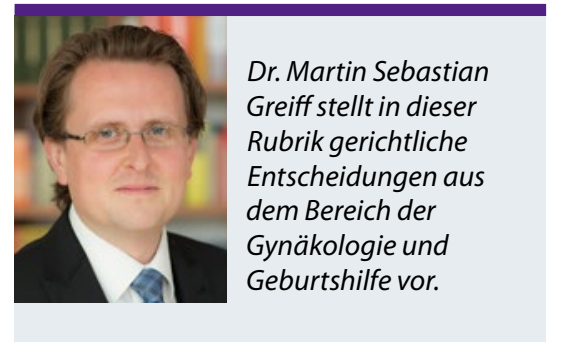

aber keine Garantie für ein gesundes Kind geben könne. Weder Gericht noch Sachverständiger beanstandeten dies. Im Gegenteil wäre es aus sachverständiger Sicht medizinisch ex ante problematisch gewesen, nun eine Amniozentese zu empfehlen. Da die Patientin später sogar selbst angab, nach einer solchen gefragt zu haben und der Beklagte in obigem Sinne geantwortet habe, ließ sich letztlich auch kein Aufklärungsdefizit mehr annehmen.

\section{Was bedeutet das Urteil für den klinischen Alltag?}

Nicht alles, was aus Patientensicht in der Medizin möglich erscheint, ist tatsächlich auch immer indiziert. Als behandlungsfehlerhaftes Unterlassen kann jedoch nur gerügt werden, wenn eine medizinisch indizierte Maßnahme unterbleibt. Natürlich darf bei einer Patientin ansonsten in der Beratung keine Fehlvorstellung hervorgerufen werden und sie muss auch immer vollständig über die Situation und alle Untersuchungsmöglichkeiten informiert werden. Da sich auch insoweit vorliegend aber letztlich keine Defizite feststellen ließen, waren die Anträge auf Schmerzensgeld und Schadensersatz der Eltern unbegründet.

Dr. Martin Sebastian Greiff, Mag. rer. publ. Rechtsanwalt und Fachanwalt für Medizinrecht, Partner der Kanzlei Ratzel Rechtsanwälte

Romanstraße 77, 80639 München

www.ratzel-rechtsanwaelte.de 